\title{
CHARACTERIZATION OF A MICROMACHINED ELECTRICAL FIELD-FLOW FRACTIONATION ( $\mu$-EFFF) SYSTEM
}

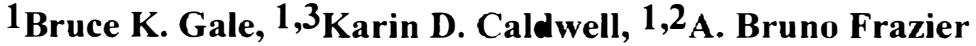 \\ ${ }^{1}$ Department of Bioengineering, ${ }^{2}$ Department of Electrical Engineering, ${ }^{3}$ Department of Chemistry \\ University of Utah, Salt Lake City, UT 84112
}

\begin{abstract}
This work introduces a $\mu$-EFFF system with an integrated conductivity detection scheme. Critical parameters in the $\mu$-EFFF system are examined including the steric transition point, system time constants, time to equilibrium, and the effects of flow rate and applied voltage on band broadening and resolution. In addition, the design, fabrication, and testing of an on-chip conductivity detector is described. The critical parameters for the on-chip conductivity detector are compared to an off-chip detector. System improvements including a streamlined channel geometry and a refined interface technique are demonstrated. Electrical time constants were reduced to the point where alternating fields may be possible. Plate heights reduced significantly with miniaturization of the system, especially with the addition of an on-chip detector. Resolution using the off-chip detector was comparable to that for macro-systems but the analysis time is onethird that of macro systems. Resolution using the on-chip detector was five times better than for the off-chip detector with an additional $50 \%$ reduction in analysis times.
\end{abstract}

\section{INTRODUCTION}

Electrical Field- Flow Fractionation (EFFF) was first described in the mid-60's, but a functional system was not built until 1991 [1]. The system was found to be excellent for the separation of colloids and other suspensions. Recently, our lab announced the development of a miniaturized EFFF system fabricated using micromachining technologies [2]. EFFF theory suggests that miniaturization of the system will improve the resolution and separation capabilities of the system by increasing the effective field realized in the channel.

Electrical Field-Flow Fractionation relies on an electric field perpendicular to the direction of separation as shown in Figure 1. The separations are performed in a low-viscosity liquid (typically an aqueous buffer solution) which is pumped through the separation channel. EFFF controls the relative velocity of particles by forcing particles towards the wall of the channel. Particles with high charge density pack closer to the wall while particles of lower charge density form a more diffuse cloud. Since flow in the channel is laminar and therefore parabolic, the particles move through the channel at differential rates based on charge and particle size. Since particle size can be determined using other

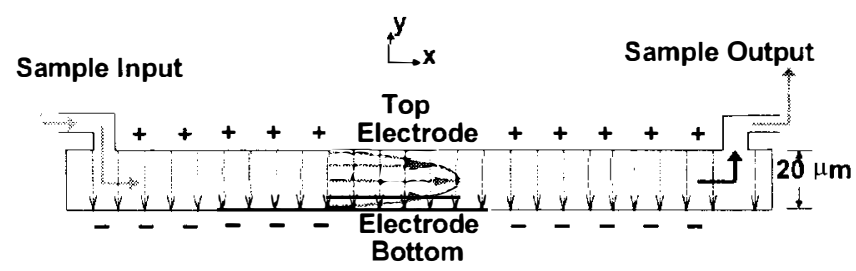

Figure 1. Schematic diagram of the operation of an EFFF system. techniques, the effect of the EFFF process is to separate particles by charge.

EFFF allows collection of fractions and has the ability to perform separations on cells. large molecules, colloids, emulsions, and delicate structures such as liposomes: separations that may be difficult or impossible in other separation systems. Applications of EFFF include cell and organelle separations, characterization of emulsions, liposomes, and other particulate vehicles, quick and accurate separations of macromolecules, environmental water monitoring, and tests for sample contamination. EFFF systems also find application in sample pretreatment.

In the last several years, great progress has been made in the fabrication of micro-scale separation systems. A myriad of successful micromachined systems of all types have been demonstrated. These publications detail several advantages found in miniaturized separation systems including increased resolution. reduced separation time. smaller sample size, and increased manufacturing precision. EFFF systems also improve when scaled into the micromachining domain.

Current macro EFFF systems are constructed using slab conductors with a thin, patterned mylar sheet between them [1]. The mylar acts as a dielectric spacer and defines the separation channel. Typical macro systems have a length of 3() to 6() $\mathrm{cm}$. a height of $127 \mu \mathrm{m}$ or greater and a height of about $2 \mathrm{~cm}$. Some experimentation was done with smaller channels. but very little has been published on the subject. Our recently published development of micromachined channels produced systems that were $6 \mathrm{~cm}$ in length, $8 \mathrm{~mm}$ in breadth, and $20 \mu \mathrm{m}$ in height [2].

\section{THEORY}

The EFFF channel, as shown in figure 1 . is a thin channel of rectangular cross-section with an aspect ratio over $8($ ). (The high aspect ratio is required to approximate infinite parallel plates.) In general, the theory behind FFF systems is well developed 13]. Understanding the theory behind EFFF is critical in understanding the operation and applications of EFFF systems and in determining the advantages created by miniaturizing the system. Several parameters of the EFFF system are expected to change with the channel height including plate height, steric transition point, the time for samples to equilibrate in the channel, electrical time constants, and effective fields in the channel.

In EFFF, an electric field is used to control the average velocity of the particles in the channel by controlling the average distance an exponentially distributed cloud of particles protrudes into the flow stream with respect to the top and bottom surfaces of the channel. If the electric field is applied as shown in figure 1. particles with higher charge will reside closer to the wall of the channel than particles with a lower charge. The particle cloud protrudes less into the flow stream and therefore has a lower velocity than particles in the middle of the stream. The difference in average velocity produces the separation. The equation for the resolution. $R_{s}$. of an EFFF system is shown in equation 1 . where 


$$
R_{s}=\frac{\frac{\Delta d}{d}}{8 D w} \sqrt{\frac{L \mu^{3} V_{e f f}^{3}}{6<v>}}
$$

$\Delta d$ is the difference between the diameters of the two particles, $\mathrm{d}$ is the average diameter of the particles, $D$ is the average diffusion coefficient of the particles. $w$ is the plate separation or channel height, $\mu$ is the electrophoretic mobility of the particles, $V_{\text {eff }}$, is the effective voltage across the channel. $L$ is the channel length, and $\langle v\rangle$ is the average buffer velocity. Examination of the equation reveals why miniaturization of the system is expected to be advantageous. The channel height, $w$. is inversely proportional to the resolution indicating that the channel dimension should be minimized. Since all the other parameters are either impossible to change or give undesirable consequences (longer run times or electrolysis), channel height reduction seems the only answer to improving EFFF systems. The question then becomes whether reducing $w$ will have the desired effect on operation of the EFFF svstem.

The effective voltage. $V_{\text {eff }}$ is a critical term that is unfortunately difficult to measure. The typical estimate of field strength made by dividing the applied voltage by the electrode separation distance, $w$, has been shown to be inconsistent with actual EFFF operation. Previous results have shown that the effective voltage is typically about 0.5 to $1 \%$ of the applied voltage depending on the makeup of the buffer $[1,4]$. This loss of effective voltage is caused by a double layer of ions that build up at the interface of the buffer and the electrode. If the double layer grows so large as to fill the separation channel, no retention occurs, the effective voltage essentially falls to zero, and the separations are destroyed. This phenomenon thus limits the ionic strength of the buffering solutions employed in the channel. A concern with miniaturizing the EFFF system is that the double layer will fill the channel even at low ionic strengths. In this case there is no advantage gained by reducing the dimensions of the channel. In our initial report of the $\mu$-EFFF system. examples of scparations were given indicating that the effective voltage does not go to zero, but the changes in effective voltage were not characterized. The effective voltage in the channel during a separation can be found using calibration techniques in which known particles are retained in the system and the required effective voltage for the observed level of retention can be calculated.

Another effect encountered in EFFF systems is the steric transition point where particle elution times begin to reverse once the radius of the particles being separated exceeds a certain length. In normal EFFF modes, smaller particles will elute before larger particles. Beyond the steric transition point, the larger particles will elute before the smaller ones. Since the steric transition point is related to the channel thickness we expect miniaturization of the

$$
d_{1}=\sqrt{\frac{2 k T_{w}}{3 \pi \eta \mu V_{c f f}}}
$$

system to impact the steric transition point and that determination of the steric transition points will give us information regarding the effective field in the channel. The steric inversion diameter, $d_{i}$. is given in equation 2 where $k$ is the Boltzmann constant, $T$ is the absolute temperature, and $\eta$ is the fluid viscosity [5].

Important characteristics of any separation system are the peak broadening and resolution of the system. which are both measured in terms of plate heights. The plate height, $H$. is a measure of variance created by the system while the particles move through the channel. The total plate height is the sum of several contributing factors. One group of factors. known as instrumental factors, $H_{i}$, can be minimized by good instrument design and operation procedures. A second group of factors are the non-equilibrium effects, $/ I_{n}$. These non-equilibrium effects are caused by the natural distribution of the sample over volume elements and the slow movement of particles between volumes. The plate height. $H$, is then given in equation 3. $H_{n}$ can be found

$$
\begin{aligned}
& H=H_{n}+H_{1} \\
& H_{n}=\frac{\mathrm{I}}{105} \frac{w^{2}\langle v\rangle}{D}
\end{aligned}
$$

using equation 4 if non-polar samples are used |3].

Experimentally, plate heights. $H$. can be measured and estimates of $H_{n}$ and $H_{i}$ can be made. Combining equations 3 and 4 , we find that plate heights should increase linearly with flow rate and the $y$-intercept on the plot is equivalent to the instrumental broadening. $H_{i}$, which we are seeking to minimize.

One area in which miniaturization is expected to improve the EFFF system is the area of sample equilibration and the need for stop flow. The establishment of equilibrium in the channel is not instantaneous and requires a relaxation time, $\tau$. equal to the time required for a particle to migrate from one electrode to the other in the presence of the applied electric field. The relaxation time, $\tau$. can be found using equation 5 .

$$
\tau=\frac{w^{2}}{2 \mu l_{i f}^{\prime}}
$$

\section{EXPERIMENTAL METHODS}

Several prototype $\mu$-EFFF systems whose fabrication was similar to that described in earlier communications, were used in this study [2]. Most of the changes in fabrication were designed to reduce peak broadening in the system. First, the input ports were reduced in size to $200 \mu \mathrm{m}$ on a side. Second. the electrical connection was not made through the input and output ports, but rather the metallization that comprised the electrodes was extended to the edge of the silicon wafer where the connection was then made. Third, angled input and output flow regions were added to the channel. Fourth, a conductive adhesive was used to bond a wire directly to the wafer in the area of the electrode metallization to allow electrical contact. Fifth. the interface between the macro and micro fluid regimes was altered. $\Lambda$ plastic ferrule from Upchurch Scientific was bonded using a UV adhesive to the surface of the silicon wafer directly over the input and output ports. The ferrule was used to localize the $125 \mu \mathrm{m}$ inner diameter stecl tubing bonded in place over the input and output ports. The $\mu$-EFFF devices were $5.4 \mathrm{~cm}$ in length, $6 \mathrm{~mm}$ in width. and with a plate separation distance of $28 \mu \mathrm{m}$. These dimensions give a void volume of $9.072 \mu \mathrm{L}$. The layout of the channel is shown in figure 2 .

Two detectors, an on-chip and an oft-chip. were demonstrated and compared with respect to the EFFF system performance. The off-chip detector was a UV detector monitoring at a wavelength of $254 \mathrm{~nm}$. The on-chip detector was a conductivity detector that was fabricated directly in the channel for detection of particles before they exit the EFFF channel. The detector consists of two parallel wires on the glass and silicon water surfaces that were fabricated in the exact same manner as the 
Output Port

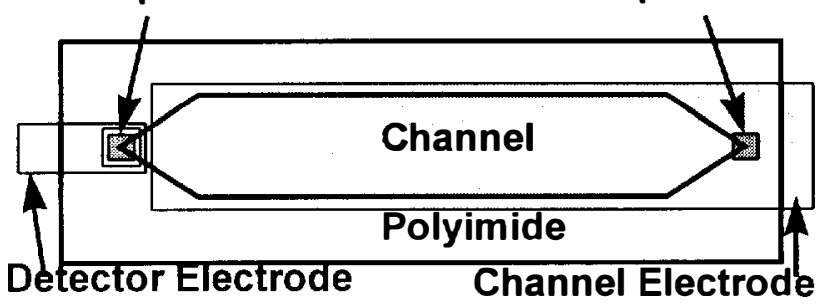

Figure 2. Configuration of channel and detector electrodes in relation to channel.

EFFF channel electrodes. Electrical connection to the detector was made as described above for the electrodes. The relationship between the detector and the channel is shown in Figure 2. The conductivity detector operated by applying a constant $5 \mathrm{~V}$ across the channel then measuring the current in the circuit.

\section{RESULTS}

Several of the earlier problems with micromachined systems were solved by the changes mentioned earlier. The dead volume in the connections to the channel was reduced to $7.7 \mu \mathrm{L}$ from the earlier systems $55 \mu \mathrm{L}$. At a flow rate of $1 \mathrm{~mL} / \mathrm{hr}(0.174 \mathrm{~cm} / \mathrm{s}$ in the channel) this reduces the delay to $27 \mathrm{sec}$ in our channel.

The time constant for changes in the electric current in the channel and by association the internal electric field was measured using a voltage step test as shown in Figure 3. The data from this test indicates that the time constant for the channel is about 4 seconds, or an order of magnitude less than that reported for a macro system [4]. Though this may still be too slow for a quickly changing field, the possibility of using a variable field is now much closer to reality.

To perform the plate height measurements, $100 \mathrm{~nL}$ samples of acetone were injected into the system for a series of flow rates. The band broadening calculations were performed using equations 3-4 and the results plotted to determine the instrumental component of the plate height. Of particular interest is the difference in band broadening between the system with the onchip detector and the off-chip detector. The results of band broadening calculations for the $\mu$-EFFF system are shown in figure 4. For comparison, the results are shown with typical band broadening calculations for current macro FFF systems and earlier reported results for a micromachined system [2]. Figure 4 shows that thinner channels produce smaller peak broadening only if the instrumental band broadening is small. The instrumental band broadening is indicated by the $y$-intercept on the graph. Note the great improvements made in band broadening by improving the macro-to-micro interfaces and switching to an on-chip detector. One effect not shown here is the influence that diffusion has on peak broadening in the off-chip detector. Figure 5 shows the peak broadening measurements with the off-chip detector. The increasing values for low flow rates indicate that diffusion is beginning to dominate at the low flow rates. Since this effect disappears with the on-chip detector. it is apparent that the diffusion must occur in the detector itself. The reason for the change in the relative importance of diffusion is due to the large dimensions found in the detector compared to the channel itself. Thus in miniaturized separation systems it appears that a requirement of the system will be on-chip detection.

The necessary relaxation time for the polystyrene particles used in these tests is less than two seconds for channels up to 30

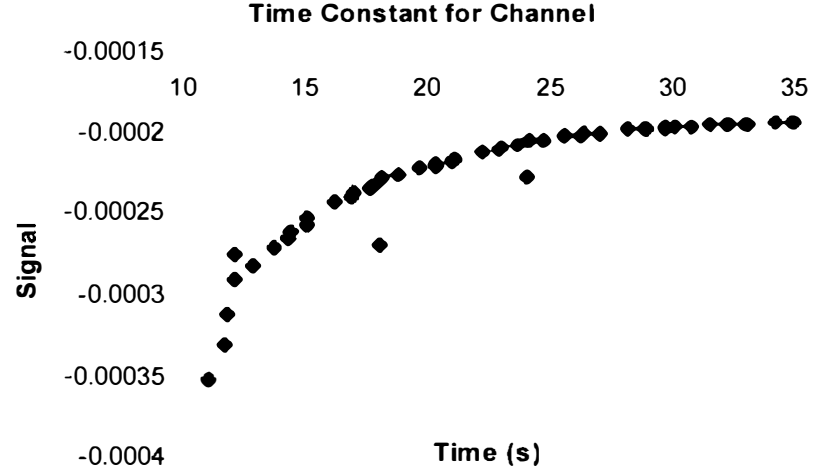

Figure 3. Step test for channel giving time constant of 4 seconds

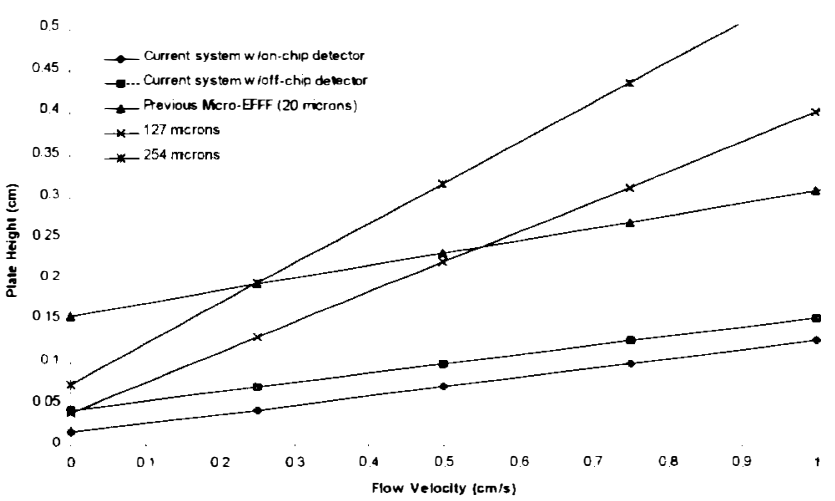

Figure 4. Plate height comparison between systems described here with both on and off chip detection, previously reported micromachined EFFF systems and two sizes of macro systems, 127 $\mu \mathrm{m}$ and $25+\mu \mathrm{m}$ channel. Note three-fold improvement over the previously reported values for a micromachined system when using the off chip detector and the five-fold improvement for the on-chip detector.

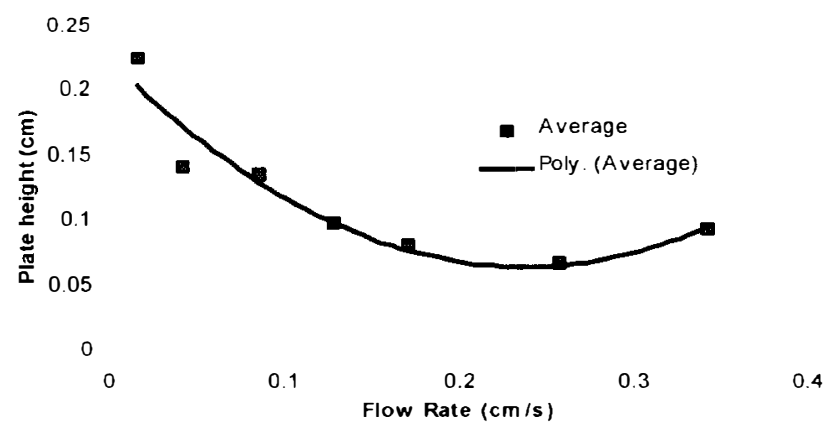

Figure 5. Plate heights for channel using off-chip detector for a series of flow rates

$\mu \mathrm{m}$ in height. This value compares to the relaxation time in the macro systems of 45 seconds and up. Our results indicate that separations can be performed without any stop time with little loss of resolution or they can be performed with a short stop time $(\sim 10$ s) to allow equilibration and an improvement in separation. This time is much less than the 5-10 min typical of macro systems.

Figure 6 shows the change in elution time as particle size increases. Note the transition from increasing elution times to decreasing elution times as a function of particle size that indicates 


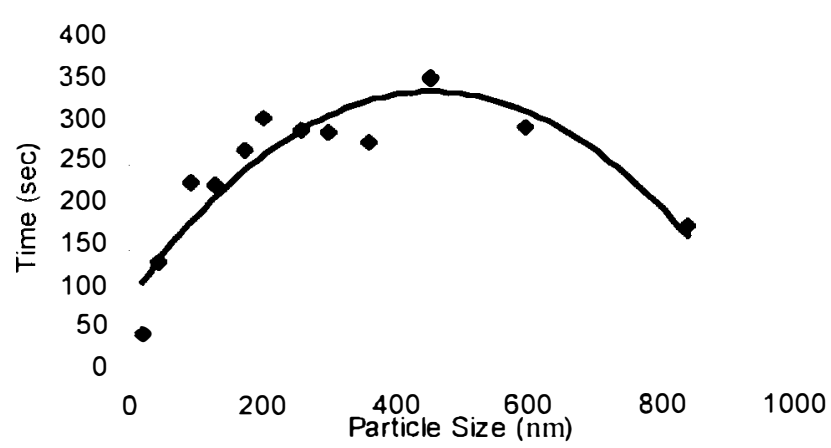

Fïgure 6. Graph of elution time for increasing particle diameter showing steric transition at about $450 \mathrm{~nm}$. Flow rate was 0.6 $\mathrm{mL} / \mathrm{hr}$ with a current of about $145 \mu \mathrm{A}$

the steric transition. The steric transition appears to occur at about $450 \mathrm{~nm}$ for this low field strength. The transition point gives a measure of the thickness of the particle cloud and which is important for determining the optimum separation conditions. Also, the data indicates that the fields are comparable to those for macro systems indicating little danger of finding effective fields too small to perform separations.

Of great importance in determining the value of a miniaturized EFFF system is an understanding of changes that occur in the effective field as the channel dimensions decrease. The effective field measurements were made as described in the theory section. A separation with the off-chip detector is shown in Figure 7. Initial measurements indicate that the effective field is from $0.38 \%$ to $0.55 \%$ of the applied field, depending on flow rate, indicating that during the scale down, some loss in effective field occurs. Reported effective field values for macro systems are generally about I \% of the applied field. Even with the apparent reduction in effective field with miniaturization, the resolution should still increase 5-fold at these values for effective field. For example, the resolution shown in figure 7 is similar to that achievable by a macro system, but the analysis time for the macro systems is at least 3 times as long. A possible explanation for loss in the measured effective field not related to miniaturization is the change in electrode materials. Gold was used for these electrodes while most macro systems use graphite. Further study of possible material effects will be studied in the future.

Figure 8 shows a separation performed using the on-chip detector. Notice the $50 \%$ reduction in run time compared to the off-chip detector. While there is significant noise in the unfiltered signal. notice the sharp peaks indicating the void peak and the various particles. The cause of the double peaks is unknown, but may be an artifact of the injection system. The resolution of the system using the double peaks as one peak is still 5 times that of the separation in figure 7 with the off-chip detector. If the double peak is eliminated, then resolution will be 10 times higher than with the off-chip detector. With some additional filtering and improvement in the measurement system, we anticipate that the on-chip detector will provide a higher performance. lower cost replacement for the bulky and expensive off-chip detectors

\section{CONCLUSION}

A $\mu$-EFFГ system was characterized with regard to changes in electric licld and plate height with channel height. The steric transition point, system time constants, time to equilibrium, and dead volumes were also measured. In addition. the design. fabrication. and testing of an on-chip conductivity detector was

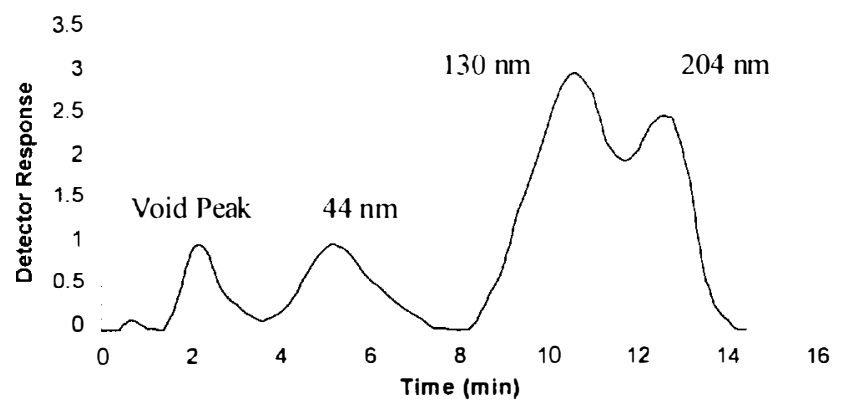

Figure 7. Separation with off-chip detector showing separation of 44. 130 , and $204 \mathrm{~nm}$ particles. Flow rate was $06 \mathrm{~mL} / \mathrm{hr}$ with a current of $1^{7} \cap \mu A$.

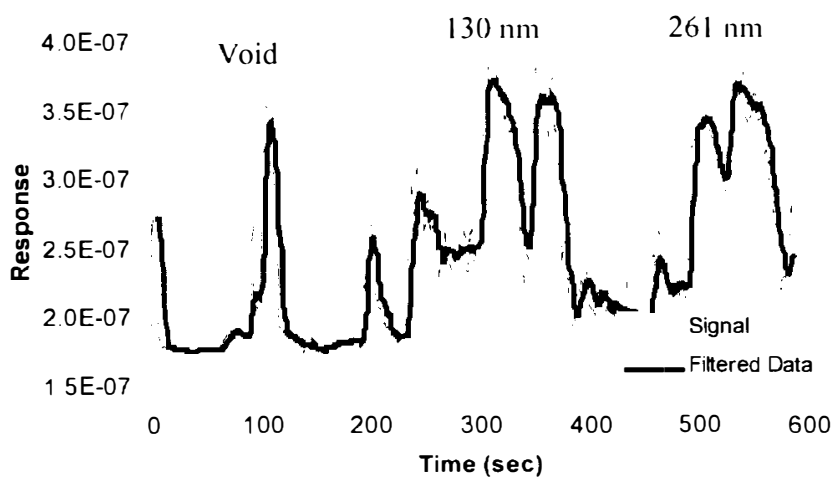

Figure 8. Separation using on-chip detector with 130 and $261 \mathrm{~nm}$ particles. The flow rate was $0.6 \mathrm{~mL} / \mathrm{hr}$ with a current of $115 \mu \mathrm{H}$.

described. Comparison was made between on and off chip detectors and the on-chip detector was shown to be superior in terms of plate heights.

\section{ACKNOWLEDGMENTS}

The authors would like to acknowledge support from Amoco Chemical Corp., a National Science Foundation Graduate Research Fellowship. and the Whitaker Foundation.

\section{REFERENCES}

1. K. D. Caldwell and Y. S. Gao, "Electrical field-flow fractionation in particle separation. Monodisperse standards," Anal. Chem., 65, 1764-1772, (1993).

2. B. K. Gale, K. D. Caldwell, and A. B. Frazier, "A Micromachined Electrical Field- Flow Fractionation System" IEEE-TBE, accepted.

3. J. C. Giddings. and K. D. Caldwell, "Field Flow Fractionation" In Physical Methods of Chemistry. Vol IIIB. B. W. Rossiter. I. F. Hamilton. Eds, New York: John Wilcy \& Sons, (1989).

4. S. A. Palkar and M. R. Schure. "Mechanistic Study of Flectrical Field Flow Fractionation. I. Nature of the Internal Field," Anal. Chem., 69, 3223-29, (1997)

5. M. N. Meyers and J. C. Giddings, "Properties of the Transition from Normal to Steric Field-Flow Fractionation." Anal. Chem., 54. 2284-89. (1982). 